
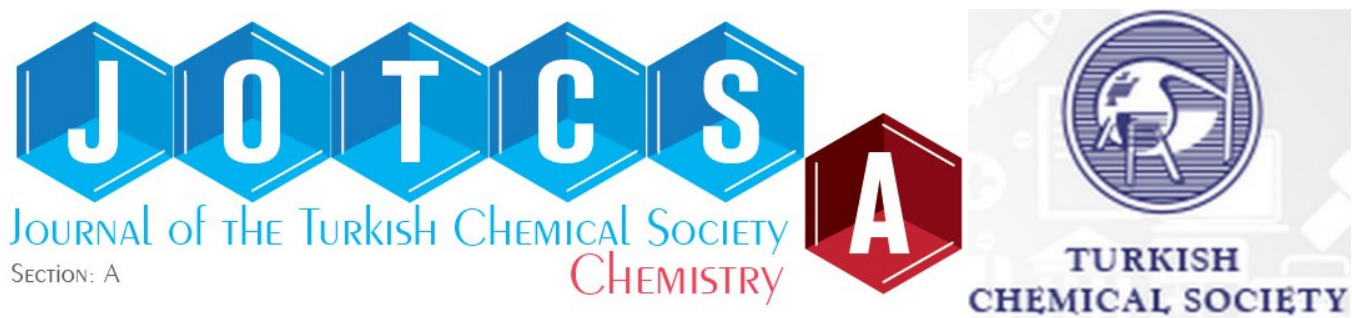

\title{
The Evaluation of Syngas Loss from Disposable Syringe Sampling System: A Case Study
}

\author{
Gábor Nagy ${ }^{1} \triangle D$, Mária Ambrus ${ }^{2}$ D \\ ${ }^{1}$ University of Miskolc, Faculty of Materials Science and Engineering, Institute of Energy and Quality, 3515, \\ Miskolc, Hungary. \\ ${ }^{2}$ University of Miskolc, Faculty of Earth Science and Engineering, Institute of Raw Material Preparation and \\ Environmental Processing, 3515, Miskolc, Hungary.
}

Abstract: The main advantage of standard gas sampling methods is their capability to keep a consistent gas composition from sampling to the analysis. On the other hand, the disadvantages include the price and possible fragility of the sampling equipment and the speed of sampling. Thus, a new tool was required with low cost and fast sampling during fieldwork. Disposable plastic syringes fulfill all these requirements with the additional advantage of applicability for multiple samplings. With the utilization of 3-way stopcocks, a filter media, and a butterfly needle, a simple but efficient sampling system was prepared. Even though the holding time of specific components is not as high as it is for a standard sample holder, the precise analysis of the gas samples is possible. After 3 hours of holding time, the concentration decrease was $0.4 \% \mathrm{CO}$, $1.6 \% \mathrm{CO}_{2}, 8.0 \% \mathrm{H}_{2}, 0.2 \% \mathrm{CH}_{4}, 0.5 \% \mathrm{C}_{2} \mathrm{H}_{4}, 0.8 \% \mathrm{C}_{2} \mathrm{H}_{6}, 3.5 \% \mathrm{H}_{2} \mathrm{~S}$ (3-part syringe) and $2.5 \% \mathrm{H}_{2} \mathrm{~S}$ (2-part syringe) In the article, equations are proposed for the examined components so that the measured concentrations could be adjusted based on the elapsed time from sampling. Syringes have been successfully utilized in the field of applied pyrolysis, for small and pilot-scale experiments, and can even be used in an industrial environment.

Keywords: gas sampling method, syngas, GC, disposable syringe

Submitted: November 10, 2019. Accepted: May 03, 2020.

Cite this: Nagy G, Ambrus M. The Evaluation of Syngas Loss from Disposable Syringe Sampling System: A Case Study. JOTCSA. 7(2):441-8.

DOI: https://doi.org/10.18596/jotcsa.644994.

*Corresponding author. E-mail: nagy.gabi@uni-miskolc.hu.

\section{INTRODUCTION}

As the energy demand of humanity is getting higher, the available energy resources must be used with the best possible efficiency. The most common utilization of solid fuels is energy production via thermochemical processes, which include, for example, combustion, gasification, and pyrolysis. Combustion is the most frequently used thermochemical process; however, the role of pyrolysis and gasification is predicted to increase in the future in both energy production and further branches of industry. During combustion, carbon monoxide, water, and heat are produced from the solid matter at high temperature, in the presence of oxygen to achieve complete combustion (1-3). In the case of gasification, the solid base material is turned into gaseous fuel - syngas - at high temperature and during partial oxidation. The main constituents of syngas are carbon monoxide and hydrogen (4). As a result, syngas can be widely used as a base material in the chemical industry, for energy or synthetic natural gas production as well (5). Pyrolysis can be carried out at a lower temperature than gasification, and usually in the absence of air. The final products, beside syngas, include significant amounts of liquid hydrocarbons such as pyrolysis oil and tar, as well as char that can be further utilized via combustion or gasification.

To adequately control both pyrolysis and gasification processes, the composition of the produced gas should be known. The compositions of the gases can 
vary greatly depending on the base material, the type of technology used, and the parameters of the experiment. The quantity of the following components should be monitored:

carbon monoxide (CO): the main component; the highest possible quantity should be in the syngas;

hydrogen $\left(\mathrm{H}_{2}\right)$ : the main component; the highest possible quantity should be in the syngas; - $\quad$ oxygen $\left(\mathrm{O}_{2}\right)$ : produced if there is a leakage in the system, so its determination is essential;

hydrocarbons $\left(\mathrm{C}_{\mathrm{x}} \mathrm{H}_{\mathrm{y}}\right)$ : fundamentally, the generation of methane is expected, but a high amount of ethane and ethene can be produced if the holding time in the reactor is short; their production is mostly a characteristic of pyrolysis, but their presence during gasification is disadvantageous, implying that the technology should be changed;

carbon dioxide $\left(\mathrm{CO}_{2}\right)$ : due to the addition of an oxidizing medium, partial oxidation occurs during gasification and $\mathrm{CO}_{2}$ is produced; the regulation of the quantity of the oxidizing medium can lead to the maximization of $\mathrm{CO}$ and the minimization of $\mathrm{CO}_{2}$ production;

hydrogen sulfide $\left(\mathrm{H}_{2} \mathrm{~S}\right)$ : produced from base materials with high sulfur content in the substoichiometric environment; essential to know its quantity for environmental considerations and the utilization of the gas.
Gasification and pyrolysis experiments of various base materials - coals, biomasses, wastes - had been conducted with small-scale and pilot-sized (Figure 1.) systems for many years to optimize syngas production. In the case of the lab-scale system, the tests were fast, so frequent sampling was necessary to determine if any parameters of the experiments had to be changed. On the other hand, the use of the pilot-sized system required a more significant number of samplings from several gas outlets to monitor the various technological steps.

Thus, the preparation of a sampling technique appropriate for both systems was required with the following criteria:

sampling should be fast, to monitor sudden changes in the gas composition;

the sampling system could be used not only in laboratory conditions but for in situ samplings;

it should be cheap, as a high number of samples are needed in case of further analyses;

the internal volume should be suitable to take samples of varying quantities (e.g., the produced gas quantity is small in the lab-scale system):

the gas composition should stay nearly constant in the sample holder until the analysis.
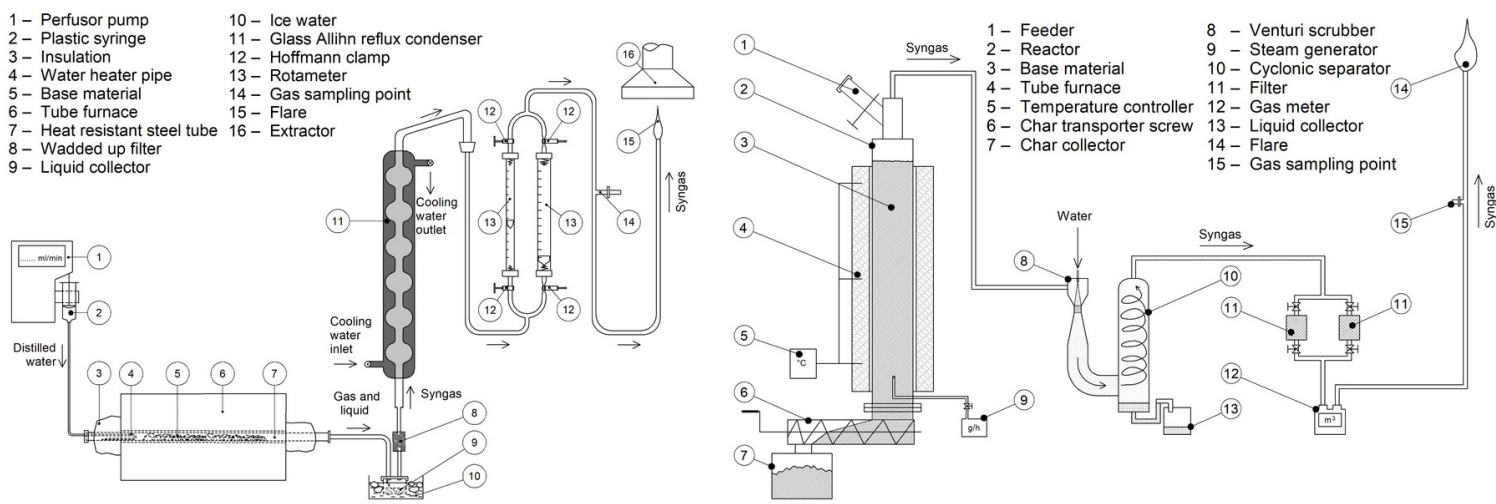

Figure 1: Lab-scale pyrolysis system (left) and pilot size gasification system (right).

The most frequent gas sampling methods (canister, sample cylinder, sampling bag, different types of sampling bulbs and gas-tight syringes) focus on holding the samples for a long time, e.g., bags for 2 days, canisters for 30 days (6). Thereby, the same gas composition is assured for the analysis as the composition during sampling. The volume of the samplers is at least $100-500 \mathrm{~cm}^{3}$, which makes the flushing and filling take several minutes, especially if the sampler is rigid. These properties of standard tools can be disadvantageous in case of experiments of smaller-scale. Glass gas-tight syringes provide fast sampling with low volume. However, they are fundamentally used for normal laboratory conditions and are not suitable for fieldwork in a dusty or contaminated environment. Furthermore, such syringes are fragile and easily blocked, rendering them single-use samplers if the immediate analysis is not possible - as it is the case for our systems. Thus, disposable plastic 3-part syringes were tested as samplers. Some of the main advantages of syringes, in contrast with the current sampling methods, are the speed of sampling, which is less than 0.5 minute, including the assembly of the sampler system and the cost, which can be a fraction of the price of the other samplers. Some examples of the approximate average retail prices of certain samplers in 2019 were as follows:

$1 \mathrm{~L}$ multi-layer foil gas sampling bag with valve and septum: 17 EUR / 19 USD;

$125 \mathrm{~mL}$ glass gas sampling bulb with stopcock and septum: 233 EUR / 269 USD; 
- $\quad 250 \mathrm{~mL}$ polypropylene gas sampling bulb with stopcock: 100 EUR / 111 USD;

- $\quad 250 \mu \mathrm{L}$ gas-tight syringe: 78 EUR / 86 USD;

I contrast, the proposed sampling system from disposable syringes would cost 1.1 EUR / 1.2 USD, with 0.6 EUR / 0.7 USD per sample using one butterfly needle and one flushing syringe.

Syringes are mainly used in the medical field. Their significance is rather low in the case of chemical analysis, where they are almost solely used as liquid feeders $(7,8)$. Furthermore, the application of disposable syringes as gas samplers has been examined in a few publications. During their experiments to analyze greenhouse gas fluxes between the soil and the atmosphere, Collier et al. (9) prepared a static chamber and collected gas samples from the chamber headspace using disposable syringes. The samples were immediately injected into vials, so there was no change in the gas composition during sampling. De Quirós et al. (10) used disposable insulin syringes to sample bubbles in the cardiovascular system of stranded cetaceans. After sampling, the contents were injected into a vacutainer. The reason to use disposable syringes was the inapplicability of gastight syringes (fragile and easily clogged) during fieldwork.

The gas holding capability of plastic syringes is scarcely tested. Cho et al. studied the air contamination of $\mathrm{CO}_{2}$-filled empty syringes with time both mathematically and experimentally. However, as the syringes were not closed off in any way, the results were not useful for the current experiment (11). On the other hand, plastic syringes filled with sulfur hexafluoride and perfluoropropane, sealed off with a plastic cap, and a 3-way stopcock were used by Ohji et al. (12), and the concentration decrease of these was examined concerning time for 24 hours. The concentration in their experiments decreased by $1-1.5 \%$ for a 3 -way stopcock and by $60-63 \%$ for the plastic cap of the syringe. According to these results, the two examined components can be stored with minimal concentration decrease in a syringe with a stopcock for one day. The investigation of the effects of syringe material (plastic and glass), storage time ( 0 and $30 \mathrm{~min}$ ) and storage temperature (0-4 and 22 ${ }^{\circ} \mathrm{C}$ ) on normal arterialized blood gas values (12 vol $\% \mathrm{O}_{2}$ and 5 vol\% $\mathrm{CO}_{2}$ ) was carried out by Knowles et al. (13). It was concluded that samples from plastic syringes should be instantly analyzed, while glass syringes should be used to store the samples.

Syringes are adequate for few-second-long medical processes. However, they are subjected to higher and longer stress during gas sampling, holding, and injection. Furthermore, these otherwise disposable tools could be reusable in case of gas sampling. After sampling, the storability of the gas is dependent on the material of the syringe and the stopcock. The barrel of the 3-part syringe is made from polypropylene, the base material of the plunger is isopropylene, the body of the stopcock is polycarbonate, and the handle is polyethylene. Among these polymers, polypropylene and polyethylene are not considered gas-tight materials, so the gas sample loss had to be examined. Moreover, if $\mathrm{H}_{2} \mathrm{~S}$ is present in the gas, it can be bound by the rubber plunger. There are some commercially available 2-part syringes, but these are difficult to obtain in large size $(50 \mathrm{~mL})$. On the other hand, 3-part syringes with rubber plunger are better sealed and provide easier plunger sliding, compared to the 2-part ones. Thus, 3-part syringes sealed with 3-way stopcocks were used for the experiments to test their performance as syngas samplers and sample holders. With high gas quantities kept in the samplers, the changes in gas composition were tested at various time intervals.

\section{MATERIALS AND METHOD}

\section{Materials}

Calibration gas from Linde was used to model raw syngas. The exact gas composition is summarized in Table 1.

Table 1: The gas mixture used to test the method.

\begin{tabular}{cc}
\hline Gases, gas mixtures & Composition, vol\% \\
\hline Hydrogen & 50 \\
Carbon monoxide & 25 \\
Carbon dioxide & 12 \\
Methane & 6 \\
Ethane & 3 \\
Ethylene & 2 \\
Hydrogen sulfide & 2 \\
\hline
\end{tabular}

Romed type 2-part and 3-part disposable plastic syringes ( $50 \mathrm{~mL})$, B.Braun type 3-way stopcocks, household cotton wool, and B.Braun type 23G scalp vein butterfly needle were used to prepare the sampling system $\left(23 \mathrm{G}=0.023^{\prime \prime}\right.$ outside diameter of the needle). The assembled gas sampling system is illustrated in Figure 2. 


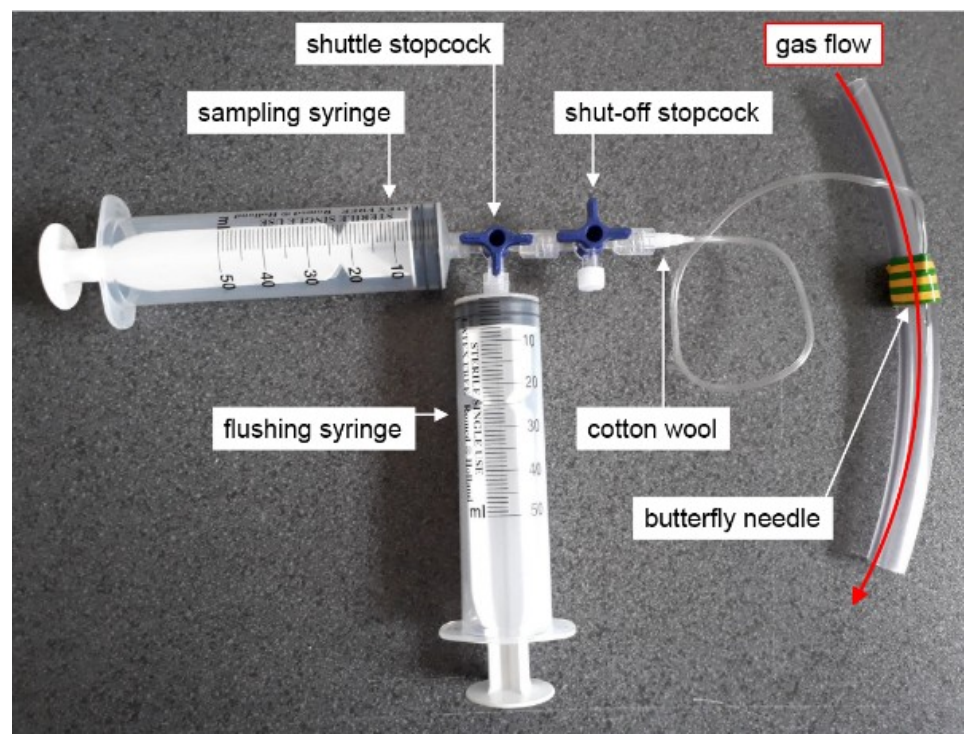

Figure 2: The gas sampling system.

\section{Methods}

The sampling method was prepared with the usage of simple medical infusion tools. Disposable syringes were used for both gas sampling and injecting the sample to a gas chromatograph for analysis. Plastic single-use syringes are available with standard conical Luer connectors (14). The two most widespread types are Luer-Slip and Luer-Lock connectors. In the case of Luer-Slip connectors, the connecting item - for example, needle, stopcock, injector, etc. - can be simply slipped in or on the cone-shaped connector. The connectors are kept together by frictional force. As the connectors can be easily disconnected, the proper connection should always be monitored. Considering that the Luer-Lock type connectors require the two connecting items to be screwed together, making disconnection significantly harder, Luer-Lock connectors were chosen.

A piece of cotton wool is used as a gas filter because of its high specific surface. It is supposed to separate materials that are either solid or able to condense and were not separated by the gas cleaning equipment. Thus, the gas chromatograph can further be protected.

Additionally, a tap or inlet is necessary where a plastic, rubber, or silicone tube can be connected to the sampler. Various sized needles were tested to connect the tube with the sampler. The trial of various types of needles revealed that using a relatively thin needle can cause many problems: the needle can bend, slip out of its plastic socket and cause injuries, get clogged and make the sampling longer because of the small inside diameter. The use of a thick needle can result in a giant hole on the tube, and, in the case of rigid tubes, gases can escape, and most of the produced components during syngas production are toxic and flammable. Thus, a 23G-25G size butterfly needle was chosen for the experiments. The tube can be pierced slantwise with the butterfly needle, just like it is done in case of blood tests or infusions, with the wings taped to the tube to keep it in position.

After assembling the sampling system, the steps of the sampling are the following (Figure 3.):

Put the shuttle stopcock in a position that allows gas to flow through all three passages (Figure 3/a.). The shut-off stopcock should be mirroring the shuttle stopcock, the passages parallel to the gas flow opened, and the perpendicular passage closed. This way, both syringes can be filled with the gas.

The tube of the butterfly needle should be flushed with the sample gas, by using one of them as a flushing syringe (Figure $3 / \mathrm{b}$.). As the sample gas enters the tube, the residing air is sucked to the flushing syringe. The total inner volume of the stopcock, the butterfly needle, and its tube is around $1.3 \mathrm{~mL}$, so approximately $10-15 \mathrm{~mL}$ gas is necessary for complete flushing.

Turning the shuttle stopcock closes the passage to the flushing syringe (Figure 3/c.), so the clean sample can only flow to the sampling syringe (Figure 3/d.).

When sampling is finished, the sampling syringe can be closed off by further turning the shuttle stopcock, opening the passage to the flushing syringe (Figure 3/e.). The gas from the flushing syringe can be injected back to the original gas flow (Figure 3/f.). Therefore it enables the user to protect themselves from the toxic components that could be released after the sampling process.

- The final step is to disconnect the empty flushing syringe and the shuttle and sampling stopcocks after the shut-off stopcock is closed (Figure 3/g.). The sample can be held in the sealed syringe that can be connected to the sampling loop of the gas chromatograph (Figure 3/h.). 


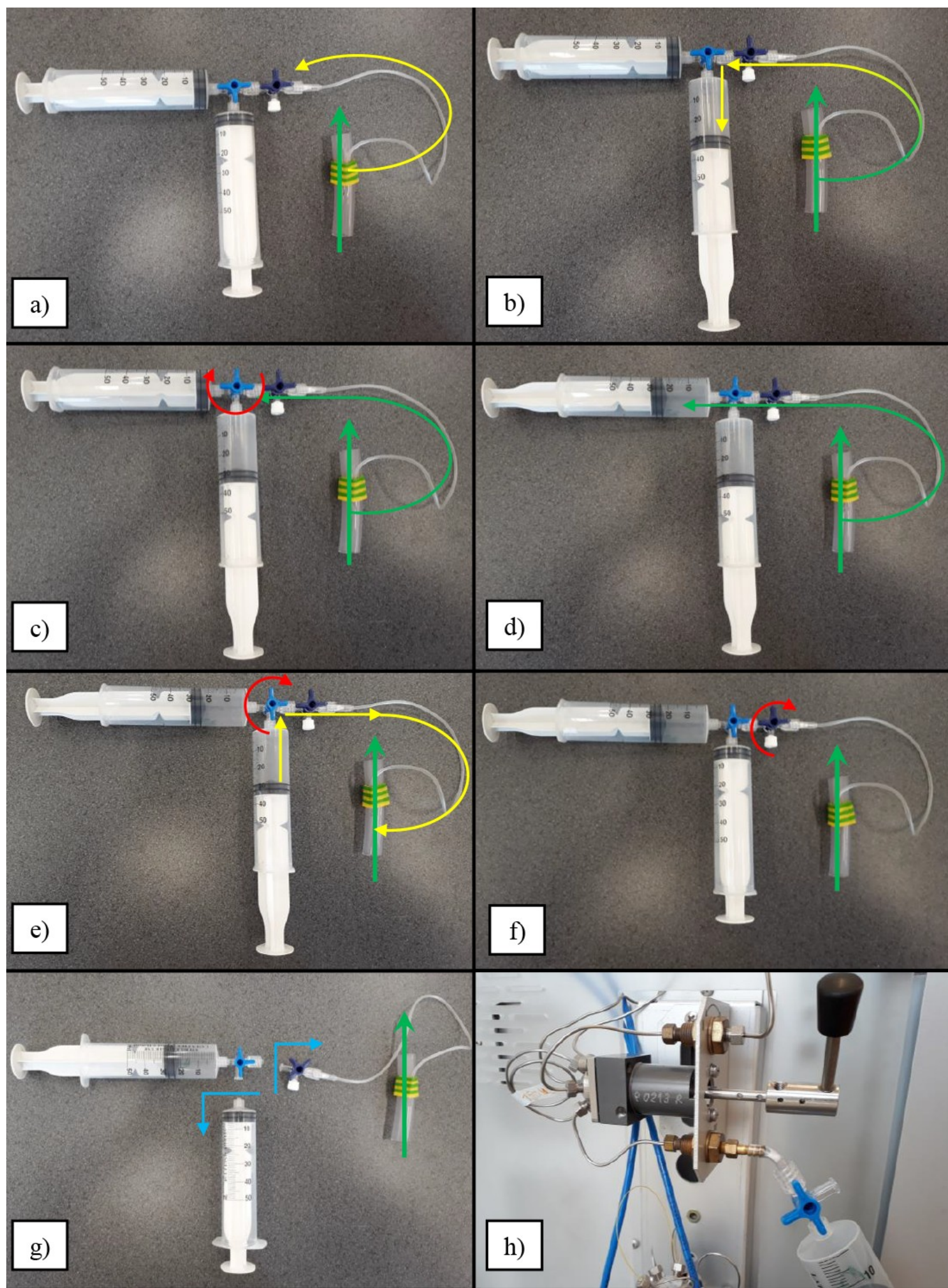

Figure 3: The operation of the sampling system.

( $a$ - connecting the sampler to the sampling point; $b$ - filling the flushing syringe with air/previous sample; $c$ - closing the flushing syringe; $d$ - sampling; e - stopping the gas sample flow; f - closing off the sampling point; $g$ - removing the sampling syringe; $h$ - attaching the sampling syringe to the gas chromatograph)

A Dani Master gas chromatograph with a TCD detector was used to test the gas holding capability of the syringes. The gas chromatograph operating conditions can be seen in Table 2 . 
Table 2: GC operation conditions.

\begin{tabular}{|c|c|}
\hline Detector & Thermal Conductivity Detector (TCD) \\
\hline Temperature & $110^{\circ} \mathrm{C}$ \\
\hline Voltage & $4 \mathrm{~V}$ \\
\hline Signal range & 1 \\
\hline Sample injection & Loop, $200 \mu \mathrm{L}$ \\
\hline Injector & $\mathrm{S} / \mathrm{SL}$ \\
\hline Mode & Splitless \\
\hline Split purge & $10 \mathrm{~mL} / \mathrm{min}$ \\
\hline Temperature & $40^{\circ} \mathrm{C}$ \\
\hline Pressure control & $400 \mathrm{kPa}$ \\
\hline Carrier gas & Helium 6.0 \\
\hline The first column ( $Q-B o n d ~ 1)$ & Restek RT-Q-Bond (Fused Silica PLOT) \\
\hline Length $\mathrm{x}$ Inner diameter $\mathrm{x}$ Film thickness & $30 \mathrm{~m} \times 0.32 \mathrm{~mm} \times 10 \mu \mathrm{m}$ \\
\hline The second column (Q-Bond 2 ) & Restek RT-Q-bond (Fused Silica PLOT) \\
\hline Length $\mathrm{x}$ Inner diameter $\mathrm{x}$ Film thickness & $15 \mathrm{~m} \times 0.53 \mathrm{~mm} \times 20 \mu \mathrm{m}$ \\
\hline The third column & Restek RT-Msieve 5A (Fused Silica PLOT) \\
\hline Length $\mathrm{x}$ Inner diameter $\mathrm{x}$ Film thickness & $30 \mathrm{~m} \times 0.53 \mathrm{~mm} \times 50 \mu \mathrm{m}$ \\
\hline Configuration of the column with software* & \\
\hline Length $\mathrm{x}$ Inner diameter $\mathrm{x}$ Film thickness & $45 \mathrm{~m} \times 0.32 \mathrm{~mm} \times 20 \mu \mathrm{m}$ \\
\hline Oven temperature & $36^{\circ} \mathrm{C}$ \\
\hline Method events & Times of switching the valve \\
\hline flow in all three columns & $0-1.80 \min$ \\
\hline flow in only the Q-Bond column & $1.80-5.70 \mathrm{~min}$ \\
\hline flow in all three columns & $5.70-12.5 \mathrm{~min}$ \\
\hline Aux gas & Nitrogen 5.0 \\
\hline Aux gas pressure & $270 \mathrm{kPa}$ \\
\hline Cycle time & $12 \mathrm{~min}$ \\
\hline Evaluation software & Clarity 3.0.6.589 \\
\hline
\end{tabular}

Samples were analyzed immediately, and 3, 6, 9, $12,18,24,36,48,59,70,95$, and 120 hours after sampling. The calibration of the instrument was checked after 9,18 , and 24 hours and corrected if necessary. Furthermore, the start-up of the instrument was always followed by calibration (before the 36-, 48-, 95- and 120-hour examination).

\section{RESULTS AND DISCUSSION}

As some experiments require much more samplings as the laboratory system can analyze at once, the period during which the samples can be used with relatively low concentration change had to be tested, because these syringes were designed to hold liquids, the composition of the gas inside changes over time as air enters the syringe. For these tests, various gas mixtures were prepared from the gases available and sealed inside the syringe with the stopcock. The gas mixtures were kept on room temperature and were not exposed to direct sunlight. The composition was analyzed at given time intervals. The signals given by the original mixture were considered $100 \%$, and the changes related to this are shown in Figure 4 . The decrease in the quantity of the components can be described with polynomials in two or three variables. Moreover, exponentials are also indicated in the figure with the coefficient of determination for each equation.

The yellow zones in Figure 4. demonstrate the range of the standard deviations of the measurements. It can be observed for all examined components, that the standard deviation of the measurements increased as the elapsed time became longer between the sampling and the measurement. Thus, the shorter the elapsed time is before measurement, the better the proposed equations are to describe the changes in composition.

In the case of $\mathrm{CO}, \mathrm{CO}_{2}, \mathrm{CH}_{4}, \mathrm{C}_{2} \mathrm{H}_{4}$, and $\mathrm{C}_{2} \mathrm{H}_{6}$, a similar trend was experienced in the concentration changes. The decrease in $\mathrm{H}_{2}$ and $\mathrm{H}_{2} \mathrm{~S}$ amount (in a 2-part syringe) showed a correlation, however, due to the better sealing ability of the syringes with rubber plunger, no further investigations were carried out about the composition changes in 2-part syringes. The evolution of the $\mathrm{H}_{2} \mathrm{~S}$ content (in a 3part syringe) shows significantly distinct characteristics. The comparison of the diagrams reveals that the decrease of hydrogen content is the most significant for approximately the first 13 hours, then the decrease in the concentration of hydrogen sulfide becomes drastically higher (in a 3part syringe). 
The reason for this phenomenon can be explained by the examination of the used syringe. Both polypropylene (barrel) and polyethylene (handle) are non-polar molecules. Hydrogen is also non-polar and has the smallest size among the examined components, so it is expected to escape at the fastest rate from the syringe (15). However, the plunger is made from isoprene, and sulfur is used for crosslinking. The crosslinking of sulfur between the double-bonds of the isoprene chains is called vulcanization (16). The quantity of sulfur affects the characteristics of the product; for example, increasing sulfur content results in higher rigidity. As the plunger is soft and able to deform, many unsaturated bonds can be found between the isoprene chains. Thus, instead of escaping to the atmosphere, hydrogen sulfide diffuses into the plunger material at a faster rate than hydrogen could escape through the wall of the syringe. The characteristic odor of hydrogen sulfide can be smelled on the plunger for several weeks after the analysis of hydrogen sulfide-containing samples, and this can, therefore, be further proven. The plunger also becomes gradually more rigid after 3050 samplings, and sliding it in the barrel also becomes difficult, as the plunger is further vulcanized due to the diffusion of sulfur. This issue can be avoided with the use of a 2-part syringe in case of hydrogen sulphide containing samples.
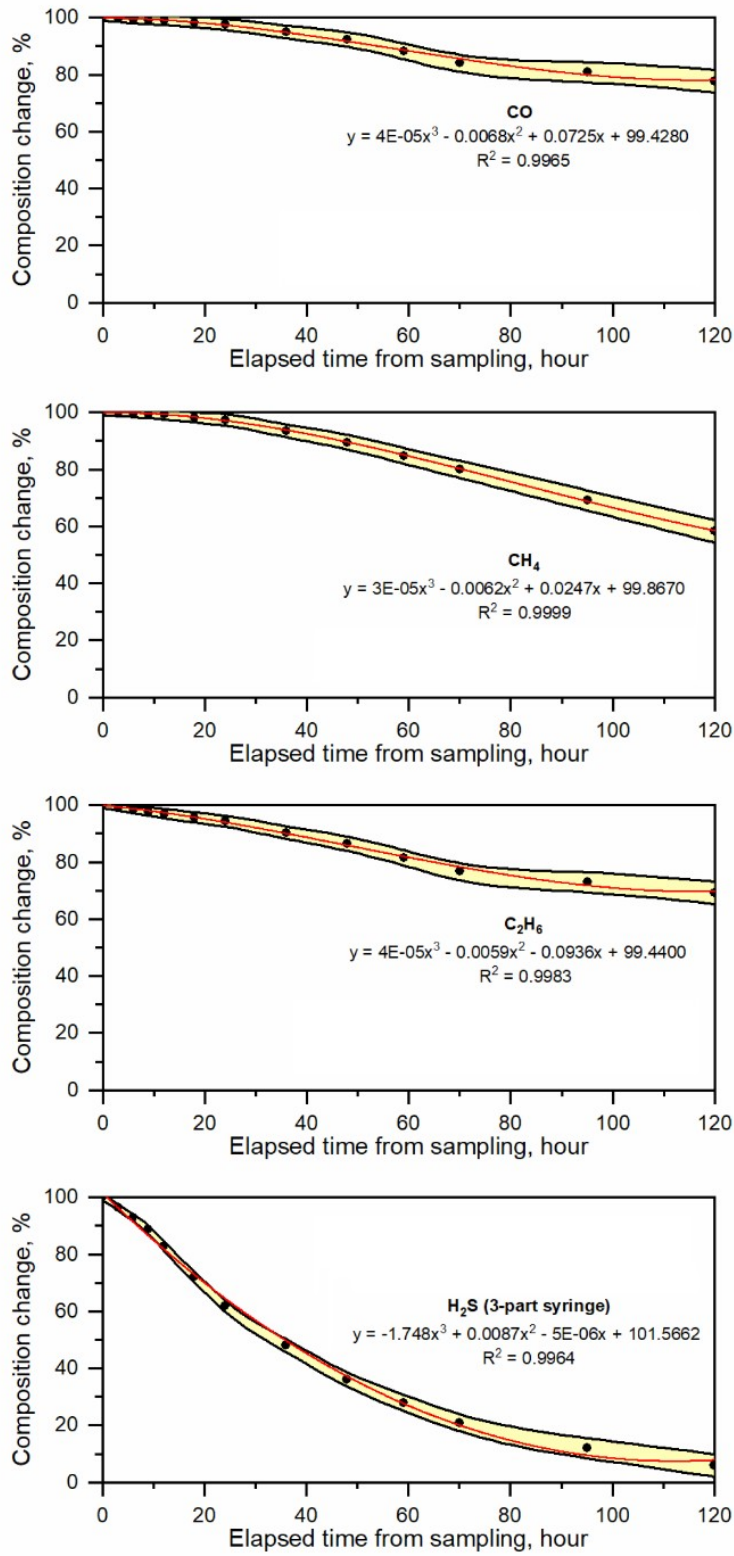

Figure 4: The changes in the composition of the calibration gas in the syringe in relation to time.

Ideally, the measurement of the stored gases should be carried out immediately after sampling as the component concentration decreases over time.
In case of the first measured time ( 3 hours), the concentration decrease was $0.4 \% \mathrm{CO}, 1.6 \% \mathrm{CO}_{2}$, $8.0 \% \mathrm{H}_{2}, 0.2 \% \mathrm{CH}_{4}, 0.5 \% \mathrm{C}_{2} \mathrm{H}_{4}, 0.8 \% \mathrm{C}_{2} \mathrm{H}_{6}, 3.5 \%$ 
$\mathrm{H}_{2} \mathrm{~S}$ (3-part syringe) and $2.5 \% \mathrm{H}_{2} \mathrm{~S}$ (2-part syringe). However, the initial concentration can be calculated using the equations given in Figure 4. Since the standard deviation for each component increases over time, the measurements should be carried out as soon as possible.

\section{CONCLUSIONS}

A new gas sampling technique for systems of various sizes was developed, using disposable plastic syringes with stopcocks and a butterfly needle, and the syngas holding capability of the syringes after sampling was examined. The hydrogen content decreases exponentially, making it one of the earliest components to leave the sample holder, along with $\mathrm{H}_{2} \mathrm{~S}$ in the 3-part syringe. Equations describing the expected gas composition related to the holding time were also proposed, making it possible to calculate the original composition and concentration of the examined gas samples. As the standard deviations of the equations also increase over time, the accuracy of the calculations decreases. Thus, the initial measurement of the samples is suggested.

\section{ACKNOWLEDGEMENT}

The described article was carried out as part of the EFOP-3.6.1-16-2016-00011 "Younger and Renewing University - Innovative Knowledge City institutional development of the University of Miskolc aiming at intelligent specialisation" project implemented in the framework of the Szechenyi 2020 program. The realization of this project is supported by the European Union, co-financed by the European Social Fund.

\section{REFERENCES}

1. Kumar V, Nanda M. Biomass Pyrolysis-Current status and future directions. Energy Sources Part aRecovery Utilization and Environmental Effects. 2016;38(19):2914-21.

2. Stiegel GJ, Ramezan M. Hydrogen from coal gasification: An economical pathway to a sustainable energy future. International Journal of Coal Geology. 2006;65(3-4):173-90.

3. Sansaniwal SK, Pal K, Rosen MA, Tyagi SK. Recent advances in the development of biomass gasification technology: A comprehensive review. Renewable \& Sustainable Energy Reviews. 2017;72:36384.
4. Basu P. Biomass Gasification and Pyrolysis Practical Design and Theory. Kidlington: Elsevier Inc.; 2010.

5. Nagy G, Wopera A, Koos T, Szabo R. The pyrolysis of canteen waste and oak mixtures in various ratios. Energy Sources Part A-Recovery Utilization and Environmental Effects. 2018;40(18):2124-36.

6. Swift J, Liss LA, Durand B. Indoor air sampling and evaluating guide. Boston: Commonwealth of Massachussetts Department of Environmental Protection; 2002.

7. Chiu G. Use of plastic syringes as measuring pipettes. Journal of Chemical Education. 1992;69(8):666.

8. Caletka R. Application of disposable plastic syringes in analytical-chemistry. Fresenius Zeitschrift Fur Analytische Chemie. 1982;311(2):124-5.

9. Collier SM, Ruark MD, Oates LG, Jokela WE, Dell C]. Measurement of Greenhouse Gas Flux from Agricultural Soils Using Static Chambers. Jove-Journal of Visualized Experiments. 2014(90):8.

10. de Quiros YB, Gonzalez-Diaz O, Saavedra P, Arbelo M, Sierra E, Sacchini S, et al. Methodology for in situ gas sampling, transport and laboratory analysis of gases from stranded cetaceans. Scientific Reports. $2011 ; 1: 10$

11. Cho DR, Cho KJ, Hawkins IF. Potential air contamination during $\mathrm{CO} 2$ angiography using a hand-held syringe: Theoretical considerations and gas chromatography. Cardiovascular and Interventional Radiology. 2006;29(4):637-41.

12. Ohji M, Adachi F, Tano Y. Sulfur hexafluoride and perfluoropropane do not escape from a plastic syringe closed with a stopcock. American Journal of Ophthalmology. 1997;123(5):709-11.

13. Knowles TP, Mullin RA, Hunter JA, Douce $F H$. Effects of syringe material, sample storage time, and temperature on blood gases and oxygen saturation in arterialized human blood samples. Respiratory Care. 2006;51(7):732-6.

14. Conical fittings with $6 \%$ (Luer) taper for syringes, needles and certain other medical equipment - Part 2: Lock fittings. Switzerland: International Organization for Standardization; 1998.

15. Karger-Kocsis J. Polypropylene - An A-Z reference: Kluwer; 1999.

16. Mark JE, Erman B, Roland M. The Science and Technology of Rubber - 4th Edition. Oxford: Academic Press; 2013. 